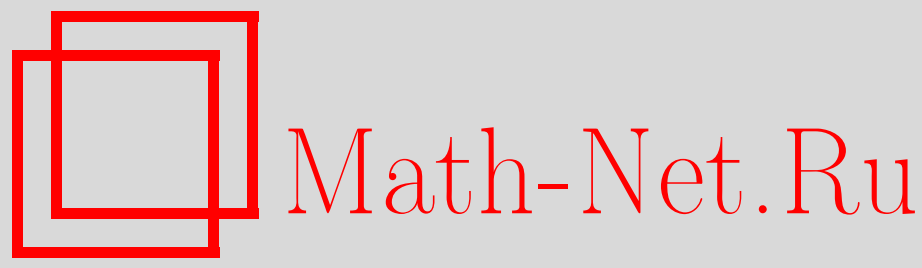

А. В. Пенской, Интегрируемые системы и топология изоспектральных многообразий, ТМФ, 2008, том 155, номер 1, 140-146

DOI: https://doi.org/10.4213/tmf6199

Использование Общероссийского математического портала Math-Net.Ru подразумевает, что вы прочитали и согласны с пользовательским соглашением http://www.mathnet.ru/rus/agreement

Параметры загрузки:

IP: 34.227 .88 .159

26 апреля 2023 г., 05:00:24

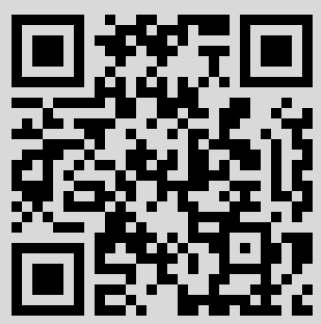




\title{
ИНТЕГРИРУЕМЫЕ СИСТЕМЫ И ТОПОЛОГИЯ ИЗОСПЕКТРАЛЬНЫХ МНОГООБРАЗИЙ
}

\begin{abstract}
Приводится краткий обзор известных результатов, касающихся исследования топологии изоспектральных многообразий с помощью интегрируемых систем. Затем излагаются новые результаты, касающиеся топологии изоспектрального многообразия матриц Якоби с нулевой диагональю. Эта топология изучается с помощью системы Вольтерра.
\end{abstract}

Ключевые слова: интегрируемые системы, изоспектральные многообразия, система Вольтерра.

\section{1. ВВЕДЕНИЕ}

Рассмотрим симплектическое многообразие $\left(X^{2 n}, \omega\right)$ и интегрируемую систему с гамильтонианом $H$ и инволютивными интегралами $F_{1}=H, F_{2}, \ldots, F_{n}$. Пусть $X_{F} \subset X$ - подмножество, заданное уравнениями $F_{1}=k_{1}, \ldots, F_{n}=k_{n}$, где $k_{i}$ - константы. Подмножество $X_{F}$ является подмногообразием для $k_{1}, \ldots, k_{n}$ в общем положении. Это подмногообразие называется поверхностью уровня интегралов. Хорошо известная теорема Лиувилля-Арнольда [1] утверждает, что если $X_{F}$ компактно и связно, то это тор. Это делает изучение топологии $X_{F}$ тривиальным. Тем не менее оказывается, что в некоторых важных примерах интегрируемых систем такое подмногообразие компактно, но его топология весьма сложна. Это происходит по причине того, что в этих примерах фазовое пространство $X$ содержит точки, в которых либо гамильтониан $H$ сингулярен, либо симплектическая форма $\omega$ сингулярна или вырожденна. В таких ситуациях теорема Лиувилля-Арнольда не применима. Тем не менее иногда можно определить соответствующий поток на всем подмногообразии $X_{F}$ и использовать его для исследования топологии $X_{F}$.

Например, это возможно, если интегрируемая система имеет представление Лакса $d L / d t=[L, A]$. Ясно, что соответствующий поток определен на всем подмногообразии $X_{F}$, которое в этом случае является изоспектральным многообразием матриц Лакса $L$, определенным уравнениями $\operatorname{tr} L^{i}=k_{i}$. Это естественным образом приводит нас к идее исследования топологии изоспектральных многообразий матриц

* Независимый московский университет; Московский государственный технический университет им. Н.Э. Баумана, Москва, Россия. E-mail: penskoi@mccme.ru 
Лакса $L$ с использованием интегрируемых потоков $d L / d t=[L, A]$. Оказывается, что в некоторых примерах топология этих изоспектральных многообразий достаточно интересна, чтобы ее изучать.

\section{2. ТОПОЛОГИЯ ИЗОСПЕКТРАЛЬНОГО МНОГООБРАЗИЯ МАТРИЦ ЯКОБИ}

Первый пример интересного спектрального многообразия был описан Томеи в 1984 г. в работе [2], где была исследована топология изоспектрального многообразия $J_{n}(n \times n)$-матриц Якоби (т.е. вещественных трехдиагональных симметрических матриц)

$$
\left(\begin{array}{ccccc}
a_{1} & b_{1} & 0 & \ldots & 0 \\
b_{1} & a_{2} & b_{2} & \ddots & \vdots \\
0 & b_{2} & \ddots & \ddots & 0 \\
\vdots & \ddots & \ddots & \ddots & b_{n-1} \\
0 & \ldots & 0 & b_{n-1} & a_{n}
\end{array}\right) .
$$

Это поверхность уровня интегралов (незамкнутой) цепочки Тоды

$\dot{a}_{i}=2\left(b_{i}^{2}-b_{i-1}^{2}\right), \quad i=1, \ldots, n, \quad \dot{b}_{i}=b_{i}\left(a_{i+1}-a_{i}\right), \quad i=1, \ldots, n-1, \quad b_{0}=b_{n}=0$.

Используя поток Тоды, Томеи вычислил эйлерову характеристику этого многообразия

$$
\chi\left(J_{n}\right)=(-1)^{n+1} \frac{B_{n+1} 2^{n+1}\left(2^{n+1}-1\right)}{n+1},
$$

где $B_{k}$ обозначает $k$-е число Бернулли, определенное как в [3]. Томеи также описал структуру $\mathrm{CW}$-комплекса для фактора этого многообразия по действию дискретной группы симметрий.

Двумя годами позже Фрид обнаружил [4], что стабильная и нестабильная стратификации, определенные потоком Тоды, являются клеточными комплексами. Используя этот факт, он нашел числа Бетти этого изоспектрального многообразия.

Пусть $\pi \in S_{n}$ - перестановка. Напомним, что интервал $[i, i+1]$ такой, что $\pi(i)<$ $\pi(i+1)$, называется подъемом. Число перестановок из $n$ элементов с $k$ подъемами называется числом Эйлера и обозначается $\left\langle\begin{array}{l}n \\ k\end{array}\right\rangle[3]$. Это важный комбинаторный объект.

Фрид доказал [4], что число Бетти $b_{k}$ равно $\left\langle\begin{array}{l}n \\ k\end{array}\right\rangle$. Он также описал умножение в кольце когомологий.

Это красивое сочетание хорошо известной интегрируемой системы, нетривиальной топологии и комбинаторики делает исследование этого изоспектрального многообразия интересным. 


\section{3. ТОПОЛОГИЯ ИЗОСПЕКТРАЛЬНОГО МНОГООБРАЗИЯ МАТРИЦ ЯКОБИ С НУЛЕВОЙ ДИАГОНАЛЬЮ}

В недавнем коротком сообщении [5] были анонсированы новые результаты, касающиеся изоспектрального многообразия матриц Якоби с нулевой диагональю. Изложим эти результаты в деталях.

Рассмотрим многообразие $M_{k}$ всех $(k \times k)$-матриц с фиксированным спектром вида

$$
L=\left(\begin{array}{ccccc}
0 & c_{1} & 0 & \ldots & 0 \\
c_{1} & 0 & c_{2} & \ddots & \vdots \\
0 & c_{2} & \ddots & \ddots & 0 \\
\vdots & \ddots & \ddots & \ddots & c_{k-1} \\
0 & \ldots & 0 & c_{k-1} & 0
\end{array}\right) .
$$

ПрЕДЛОЖенИЕ 1. 1. Собственные числа $L$ имеют вид 0 (если $k$ нечетно), $\pm \lambda_{1}$, $\pm \lambda_{2}, \ldots$.

2. Если все собственные числа различны, то $M_{k}$ - компактное гладкое многообразие, и его топология не зависит от собственных чисел.

ДоКАЗАТЕЛЬСТво. Умножая все четные строки и столбцы $L-\lambda I$ на -1 , мы получим $-L-\lambda I$. Поэтому $\operatorname{det}(L-\lambda I)=\operatorname{det}(-L-\lambda I)$, откуда следует первое утверждение. Доказательство второго утверждения аналогично доказательству подобного утверждения для изоспектрального многообразия матриц Якоби, предложенному Томеи [2].

Многообразие $M_{k}$ является поверхностью уровня интегралов (незамкнутой) системы Вольтерра

$$
\dot{c}_{i}=\frac{1}{2} c_{i}\left(c_{i+1}^{2}-c_{i-1}^{2}\right), \quad c_{0}=c_{k}=0 .
$$

Обычно эта система записывается в переменных $u_{i}=c_{i}^{2}$. Хорошо известно, что система Вольтерра может быть записана в лаксовой форме $\dot{L}=[L, A(L)]$, откуда следует, что поток Вольтерра сохраняет спектр $L$, т.е. является потоком на $M_{k}$.

Система Вольтерра интегрируемая, что было доказано в 1974 г. Манаковым [6] и годом позже независимо Кацем и ван Мёрбеке [7]. Хорошо известно, что система Вольтерра является бигамильтоновой (см., например, книгу [8]).

Оказывается, что с точки зрения топологии имеются два различных случая, зависящих от четности $k$.

Пусть $P_{k}\left(c_{1}, \ldots, c_{k-1}, \lambda\right)=\operatorname{det}(L-\lambda I)$ - характеристический многочлен $(k \times k)$ матрицы $L$ вида (2). Например, $P_{2}\left(c_{1}, \lambda\right)=\lambda^{2}-c_{1}^{2}$. Мы будем говорить, что множество $J \subset\{1, \ldots, k-1\}$ является вполне несвязным, если $J$ не содержит двух последовательных целых чисел $i$ и $i+1$. Пусть $I_{0}=1$ и $I_{l}=\sum c_{i_{1}}^{2} \ldots c_{i_{l}}^{2}, l \geqslant 1$, где суммирование проводится по всем $i_{1}<\cdots<i_{l}$ таким, что множество $\left\{i_{1}, \ldots, i_{l}\right\}$ вполне несвязно. 
ПРЕДЛОЖЕНИЕ 2. 1. В четном $(k=2 l)$ случае

$$
P_{2 l}\left(c_{1}, \ldots, c_{2 l-1}, \lambda\right)=\sum_{i=0}^{l}(-1)^{i} \lambda^{2 l-2 i} I_{i} .
$$

2. В нечетном $(k=2 l+1)$ случае

$$
P_{2 l+1}\left(c_{1}, \ldots, c_{2 l}, \lambda\right)=\sum_{i=0}^{l}(-1)^{i+1} \lambda^{2 l+1-2 i} I_{i} .
$$

3. Многообразие $M_{k}$ задано уравнениями $I_{i}=k_{i}, i=0, \ldots, l$, где $k_{i}-$ константы.

ДокАзАтельство. Разлагая $\operatorname{det}(L-\lambda I)$ по последней строке и последнему столбцу, мы получим рекуррентную формулу

$$
P_{N}\left(c_{1}, \ldots, c_{N-1}, \lambda\right)=-\lambda P_{N-1}\left(c_{1}, \ldots, c_{N-2}, \lambda\right)-c_{N-1}^{2} P_{N-2}\left(c_{1}, \ldots, c_{N-3}, \lambda\right) .
$$

Используя эту формулу и тождества $P_{1}(\lambda)=-\lambda$ и $P_{2}\left(c_{1}, \lambda\right)=\lambda^{2}-c_{1}^{2}$, по индукции получаем утверждение предложения.

Заметим, что формулы для $I_{i}$ аналогичны формулам, полученным в работе [9] для периодической системы Вольтерра.

ПреДЛОЖЕНИЕ 3. 1. В четном $(k=2 l)$ случае $M_{k}$ имеет $2^{l}$ изоморфньх компонент связности. Более того, каждая компонента изоморфна изоспектральному многообразию матрии, Якоби.

2. В нечетном $(k=2 l+1)$ случае многообразие $M_{k}$ связно.

3. Многообразие $M_{k}$ компактно.

ДокАзАТЕЛЬСтво. Если $k=2 l$, то $I_{l}=c_{1}^{2} c_{3}^{2} \ldots c_{2 l-1}^{2} \neq 0$, потому что все собственные числа различны (см. предложение 1 ). Из этого следует, что $M_{k}$ имеет по крайней мере $2^{l}$ компонент, определяющихся $l$ значениями $\operatorname{sgn} c_{2 i-1}, i=1, \ldots, l$. Доказательство того, что каждая из этих компонент связна, схоже с предложенным Томеи доказательством того, что изоспектральное многообразие матриц Якоби связно.

Существует хорошо известное отображение [10], [11]

$$
a_{i}=\frac{1}{2} c_{i}\left(c_{i+1}^{2}-c_{i-1}^{2}\right), \quad b_{i}=-\frac{1}{2} c_{2 i} c_{2 i-1},
$$

переводящее решения системы Вольтерра в решения цепочки Тоды. Если $k=2 l$, то легко проверить, что это отображение - изоморфизм между каждой связной компонентой многообразия $M_{k}$ и соответствующим изоспектральным многообразием матриц Якоби. Это завершает доказательство первого утверждения.

Доказательство утверждений 2 и 3 сходно с предложенным Томеи [2] доказательством того, что изоспектральное многообразие матриц Якоби связно и компактно. 
Предложение 3 сводит изучение топологии $M_{k}$ в четном случае к исследованию уже известной топологии изоспектрального многообразия матриц Якоби, изучавшегося Томеи и Фридом. Рассмотрим только нечетный случай.

В настоящее время неясно, как вычислять группы гомологий. Стабильная и нестабильная стратификации не являются клеточными комплексами в этом случае, и невозможно применить прямой подход, подобный подходу Фрида [4]. Отметим также, что в 1992 г. Блох, Брокетт и Ратью [12] использовали идею двойного скобочного представления, чтобы показать, что цепочка Тоды является градиентным потоком. В 1998 г. мы показали [13], что поток Вольтерра также является градиентным потоком. Это естественным образом приводит к идее нахождения группы гомологий с помощью комплекса Морса. K сожалению, это невозможно, так как стабильная и нестабильная стратификации не трансверсальны друг другу. Вопрос вычисления групп гомологий в нечетном случае остается открытым, насколько это известно автору. В настоящей работе мы вычисляем эйлерову характеристику многообразия $M_{k}$, используя поток Вольтерра, так, как это было анонсировано в работе [5]. Предлагаемый подход близок к подходу Томеи [2], но комбинаторика точек равновесия потока Вольтерра и их индексов гораздо сложнее.

Пусть $K=(1 / 4) \operatorname{diag}(1,2,3, \ldots)$ и $f(L)=\operatorname{tr} K L^{2}$. Доказано [13], что (отрицательный) градиентный поток $f(L)$ относительно некоторой метрики (сконструированной в явном виде в работе [13]) совпадает с потоком Вольтерра. Выберем $\lambda_{i}$ (см. предложение 1) таким образом, что все $\lambda_{i}>0$ и $\lambda_{i}>\lambda_{i+1}$.

ПреДЛОЖЕНИЕ 4. 1. Критические точки $f(L)$ на $M_{2 l+1}$ находятся во взаимно однозначном соответствии с тройками $[j, s, \pi]$, состоящими из числа $j, 0 \leqslant j \leqslant l$, набора из $l$ чисел $s=\left(s_{1}, \ldots, s_{l}\right)$, где кажсдее $s_{i}$ равно 0 или 1, и перестановки $\pi \in S_{l}$.

2. Индекс критической точки, соответствующей $[j, s, \pi]$, равен количеству чисел $i, 0 \leqslant i \leqslant l-1$, таких, что $i \neq j, j-1 u \pi(i)<\pi(i+1)$, плюс 1 , если $j \neq l$.

ДокАЗАтЕЛЬСтво. Критические точки являются точками равновесия потока Вольтерра. Используя этот факт, а также явные полиномиальные уравнения $I_{i}=k_{i}$ многообразия $M_{k}$, можно по индукции доказать, что критические точки - это в точности такие точки, что в точности $l$ из $2 l$ величин $c_{1}, \ldots, c_{2 l}$ равны нулю, с дополнительным свойством, что если $c_{i} \neq 0$, то $c_{i-1}=c_{i+1}=0$. Исследуя спектр соответствующей матрицы, получаем описание, данное в формулировке предложения. Запишем $c_{1}, \ldots, c_{2 l}$ как набор из $2 l$ элементов: $\left(c_{1}, \ldots, c_{2 l}\right)$. Тройка $[0, s, \pi]$ соответствует критической точке

$$
\left(c_{1}, \ldots, c_{2 l}\right)=\left(0,(-1)^{s_{1}} \lambda_{\pi(1)}, 0,(-1)^{s_{2}} \lambda_{\pi(2)}, 0, \ldots,(-1)^{s_{l}} \lambda_{\pi(l)}\right),
$$

тройка $[l, s, \pi]-$ точке

$$
\left((-1)^{s_{1}} \lambda_{\pi(1)}, 0,(-1)^{s_{2}} \lambda_{\pi(2)}, 0, \ldots,(-1)^{s_{l}} \lambda_{\pi(l)}, 0\right)
$$

а тройка $[j, s, \pi]$, где $j \neq 0, l$, соответствует точке

$$
\left((-1)^{s_{1}} \lambda_{\pi(1)}, 0,(-1)^{s_{2}} \lambda_{\pi(2)}, 0, \ldots,(-1)^{s_{j}} \lambda_{\pi(j)}, 0,0,(-1)^{s_{j+1}} \lambda_{\pi(j+1)}, 0, \ldots,(-1)^{s_{l}} \lambda_{\pi(l)}\right) .
$$


Это доказывает первое утверждение. Второе утверждение может быть получено при изучении гессиана $f(L)$. Есть два способа вычисления индекса, но мы опускаем детали, так как оба способа достаточно длинные и технические. Первый способ ввести хорошие локальные координаты и вычислить индекс прямым вычислением. Второй способ следующий. Заметим, что $f(L)=\operatorname{tr} K L^{2}$ определено для любой $(k \times k)$-матрицы $L$. Идея состоит в том, чтобы рассмотреть $M_{k}$ как подмногообразие орбиты $O$ группы $G L(k)$, действующей на $(k \times k)$-матрицах сопряжением. Следует начать с изучения гессиана ограничения $\left.f\right|_{O}$, а затем перейти к $\left.f\right|_{M_{k}}$.

ПреДЛОЖЕНИЕ 5. 1. Эйлерова характеристика многообразия $M_{2 l+1}$ равна

$$
\chi\left(M_{2 l+1}\right)=2^{2 l+2}\left(2^{l+2}-1\right) \frac{B_{l+2}}{l+2},
$$

где $B_{l+2}$ - соответствующее число Бернулли.

2. Если мы положим $\chi\left(M_{1}\right)$ равной нуль, то экспоненииальная прочзводящая функиия чисел $\chi\left(M_{2 l+1}\right)$ равна $-\operatorname{th}^{2}(2 z)$, m.e.

$$
-\operatorname{th}^{2}(2 z)=\sum_{l \geqslant 0} \chi\left(M_{2 l+1}\right) \frac{z^{l}}{l !} .
$$

ДоказАТЕЛЬСтво. Обозначим число подъемов в $\pi$ через $p(\pi)$. Пусть

$$
\psi(n)=\sum_{m=0}^{n}(-1)^{m}\left\langle\begin{array}{l}
n \\
k
\end{array}\right\rangle
$$

тогда из формулы (7.56) в книге [3] следует, что

$$
1+\operatorname{th} z=\sum_{n \geqslant 0} \psi(n) \frac{z^{n}}{n !} .
$$

Используя предложение 4 , можно доказать, что эйлерова характеристика $\chi\left(M_{2 l+1}\right)$ равна

$$
\chi\left(M_{2 l+1}\right)=2^{l} \sum_{j=1}^{l-1}\left(\begin{array}{l}
l \\
j
\end{array}\right) \sum_{\pi_{1} \in S_{j}, \pi_{2} \in S_{l-j}}(-1)^{p\left(\pi_{1}\right)+p\left(\pi_{2}\right)+1} .
$$

Это выражение может быть переписано в терминах $\psi(n)$ следующим образом:

$$
\begin{aligned}
\chi\left(M_{2 l+1}\right) & =-2^{l} \sum_{j=1}^{l-1}\left(\begin{array}{l}
l \\
j
\end{array}\right)\left(\sum_{\pi_{1} \in S_{j}}(-1)^{p\left(\pi_{1}\right)}\right)\left(\sum_{\pi_{2} \in S_{l-j}}(-1)^{p\left(\pi_{2}\right)}\right)= \\
& =-2^{l} \sum_{j=1}^{l-1}\left(\begin{array}{l}
l \\
j
\end{array}\right) \psi(j) \psi(l-j) .
\end{aligned}
$$

Из этого тождества, а также тождества (4) следует второе утверждение. Используя разложение th $z$ и формулу $\operatorname{th}^{\prime} z=1-\operatorname{th}^{2} z$, мы получим первое утверждение. 


\section{4. ЗАКЛЮЧЕНИЕ}

Мы получили формулу (3) для эйлеровой характеристики изоспектрального многообразия $M_{k}$ вещественных $(k \times k)$-матриц Якоби с нулевой диагональю для нечетного $k$. Эта формула показывает, что топология $M_{k}$ довольно сложна. Например, из этой формулы можно увидеть, что многообразие $M_{5}$ является компактной поверхностью рода 5, что может показаться довольно неожиданным, потому что это многообразие определено простыми уравнениями

$$
c_{1}^{2}+c_{2}^{2}+c_{3}^{2}+c_{4}^{2}=k_{1}, \quad c_{1}^{2} c_{3}^{2}+c_{1}^{2} c_{4}^{2}+c_{2}^{2} c_{4}^{2}=k_{2}
$$

в пространстве $\mathbb{R}^{4}$ переменных $c_{1}, \ldots, c_{4}$. Очень интересно было бы получить числа Бетти $M_{k}$. Ожидается, что ответ будет включать в себя нетривиальную комбинаторику.

Интересно отметить, что эйлеровы характеристики изоспектральных многообразий матриц Якоби (1) и матриц Якоби с нулевой диагональю (3) связаны простой формулой

$$
\chi\left(J_{l+1}\right)=(-1)^{l} 2^{l} \chi\left(M_{2 l+1}\right) .
$$

Было бы интересно объяснить этот факт.

Благодарности. Автор благодарен А. П. Веселову за постановку задачи, а также О. Корнеа и А. Медведовской за плодотворные обсуждения.

\section{Список литературы}

[1] В. И. Арнольд, Математические методы классической механики, Наука, М., 1989.

[2] C. Tomei, Duke Math. J., 51:4 (1984), 981-996.

[3] Р. Л. Грэхем, Д. Кнут, О. Паташник, Конкретная математика: Основание информатики, Мир, М., 1998.

[4] D. Fried, Proc. Amer. Math. Soc., 98:2 (1986), 363-368.

[5] А. В. Пенской, УМН, 62:3 (2007), 213-214; arXiv: math-ph/0701061.

[6] С. В. Манаков, ЖЭТФ, 67:2 (1974), 543-555.

[7] M. Kac, P. van Moerbeke, Adv. Math., 16:2 (1975), 160-169.

[8] Л. Д. Фаддеев, Л. А. Тахтаджян, Гамильтонов подход в теории солитонов, Наука, М., 1986.

[9] А. П. Веселов, А. В. Пенской, Докл. РАН, 366:3 (1999), 299-303.

[10] P. A. Damianou, Phys. Lett. A, 155:2-3 (1991), 126-132.

[11] В. Л. Верещагин, Матем. заметки, 48:2 (1990), 145-148.

[12] A. M. Bloch, R. W. Brockett, T. S. Ratiu, Comm. Math. Phys., 147 (1992), 57-74.

[13] A. V. Penskoï, Regul. Chaotic Dyn., 3:1 (1998), 76-77. 